\title{
Mode of action and structure-activity relationship studies of geobacillin I
}

\author{
Neha Garg ${ }^{1}$, Trent J Oman ${ }^{2}$, Tsung-Shing Andrew Wang ${ }^{3}$, Chantal V Garcia De Gonzalo², Suzanne Walker ${ }^{4}$ \\ and Wilfred A van der Donk ${ }^{1,2}$
}

The Journal of Antibiotics (2014) 67, 133-136; doi:10.1038/ja.2013.112; published online 30 October 2013

Keywords: lanthionine; lantibiotics; lipid II; membrane disruption; mode of action; nisin

Lanthipeptides are lanthionine- and methyllanthionine-containing peptides that are ribosomally synthesized and post-translationally modified. ${ }^{1}$ Lanthipeptides that possess antimicrobial activity are called lantibiotics. ${ }^{2}$ Lanthionines consist of two alanine residues that are linked through a thioether that connects their $\beta$-carbons, and methyllanthionines contain an additional methyl group (Figure 1a). Nisin is the best studied and longest known lantibiotic and has been used as a food preservative for over 50 years. ${ }^{3,4}$ Nisin displays antibacterial activity against clinically important pathogens such as methicillin-resistant Staphylococcus aureus, vancomycin-resistant Enterococci, Streptococcus pneumonia, and food-borne pathogens such as Clostridium botulinum, and Listeria monocytogenes. ${ }^{5-7}$ Despite its use for over 50 years, reports of resistance against nisin have been scarce. ${ }^{8-12}$ The slow development of resistance may stem from the dual mode of action of nisin. Nisin exhibits antimicrobial activity by binding to the pyrophosphate moiety of lipid II (Figure 1b), ${ }^{13,14}$ a membrane-bound advanced intermediate involved in the biosynthesis of the cell wall. By doing so, nisin inhibits the transglycosylation step in cell wall biogenesis and sequesters lipid II from its functional location. ${ }^{15,16}$ Furthermore, the nisin-lipid II complex leads to the formation of pores in the membrane, causing cell death. ${ }^{17}$

Recently, we characterized two lanthipeptides, geobacillin I and geobacillin II, from the thermophilic bacterium Geobacillus thermodenitrificans NG80-2. ${ }^{18}$ Geobacillin I contains seven thioether bridges, one dehydroalanine (Dha) and one dehydrobutyrine (Figure 1c). The $\mathrm{N}$-terminal A and B rings of geobacillin I are very similar to the corresponding rings of nisin; however, the C-terminal structures are very different (Figure 1c). The nisin A and B rings are involved in lipid II binding, ${ }^{13}$ and hence we anticipated that geobacillin I might also bind lipid II. The three amino-acid linker peptide between the $\mathrm{C}$ and $\mathrm{D}$ rings of nisin has been shown to be indispensable for pore formation activity..$^{17,19-21}$ For instance, the $\Delta \mathrm{N} 20 \Delta \mathrm{M} 21$ and N20P/M21P mutants of nisin lost pore formation ability against Gram-positive bacteria. ${ }^{17,19}$ Geobacillin I has only a single amino acid between the $\mathrm{C}$ and $\mathrm{D}$ rings, similar to the $\Delta \mathrm{N} 20 \Delta \mathrm{M} 21$ mutant of nisin. Thus, based on the available data on nisin, we anticipated that geobacillin I would bind to lipid II but not form pores in the membrane of Gram-positive bacteria. In this work, we tested these expectations experimentally.

We first conducted antimicrobial activity assays in liquid medium. In these assays, geobacillin I exhibited a fourfold higher MIC against Bacillus subtilis ATCC 6633 compared with nisin (Table 1). Flow cytometry was then used to examine changes in the polarization of the bacterial membrane of B. subtilis ATCC 6633 upon incubation with geobacillin I using the membrane potential-sensitive dye $3,3^{\prime}$ diethyloxacarbocyanine iodide. ${ }^{22}$ Incubation with geobacillin I resulted in a significant decrease in the mean fluorescence intensity, similar to the observations when the same experiments were carried out with nisin (Figure 2a and Supplementary Figures S1 and S2). The unexpected ability of geobacillin I to form pores despite the single amino-acid linker between rings $\mathrm{C}$ and $\mathrm{D}$ may be a consequence of the overall differences between the C-terminal region of geobacillin I and nisin (Figure 1c).

We also investigated the efficiency of pore formation by geobacillin I using propidium iodide (PI) — a membrane impermeable fluorescent dye. Upon pore formation or membrane disruption, PI can enter the cell, resulting in an increase in fluorescence intensity because of the interaction of PI with nucleic acids. PI uptake was monitored at nine different concentrations with each experiment conducted in triplicate (Figure 2b and Supplementary Figure S3). The data showed only twofold lower efficiency in PI uptake for geobacillin I, with $\mathrm{IC}_{50}$

\footnotetext{
${ }^{1}$ Department of Biochemistry, University of Illinois at Urbana-Champaign, Urbana, IL, USA; ${ }^{2}$ Department of Chemistry, Howard Hughes Medical Institute, University of Illinois at Urbana-Champaign, Urbana, IL, USA; ${ }^{3}$ Department of Chemistry and Chemical Biology, Harvard University, Cambridge, MA, USA and ${ }^{4}$ Department of Microbiology and Immunobiology, Harvard Medical School, Boston, MA, USA

Correspondence: Professor WA van der Donk, Department of Chemistry, Howard Hughes Medical Institute, University of Illinois at Urbana-Champaign, 600 South Mathews Avenue, Urbana, IL 61801, USA.

E-mail: vddonk@illinois.edu

This study is dedicated to Professor Christopher T Walsh for his inspiring leadership in natural product biosynthesis and mechanistic enzymology.

Received 23 August 2013; revised 28 September 2013; accepted 2 October 2013; published online 30 October 2013
} 
a<smiles>[R]C(NC)C(NC(C)C)C(=O)NC(CSc1ccccc1)C(C)=O</smiles>

c

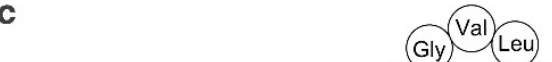

b

D-Ala

D-Ala
L-Lys
-D-Glu

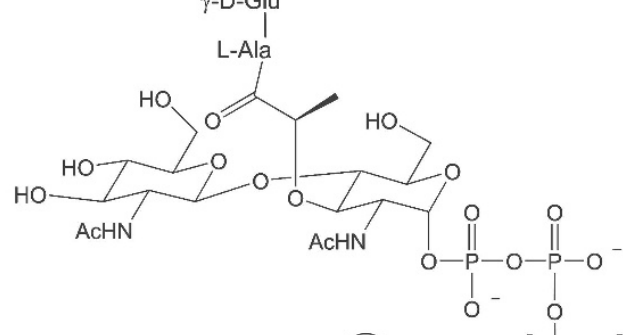

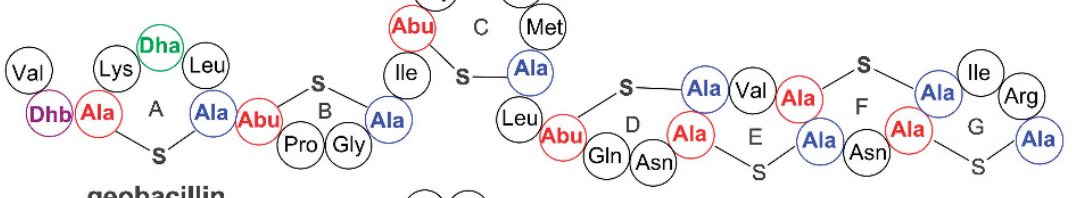

geobacillin
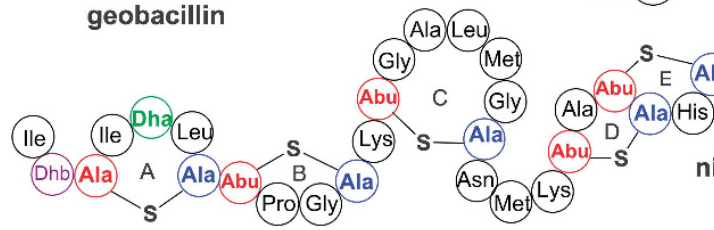

Ala His

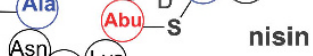

nisin

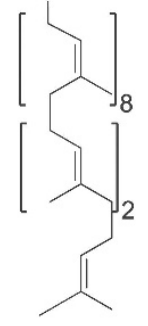

Figure 1 Structures of (a) lanthionine and methyllanthionine, (b) lipid II, and (c) geobacillin I and nisin. The shorthand notation for lanthionine (Lan) and methyllanthionine (MeLan) depicted in $\mathbf{a}$ is used in $\mathbf{c}$.

Table 1 Specific activity of nisin, geobacillin I and the geobacillin I analogs generated in this study against B. subtilis ATCC 6633

\begin{tabular}{lcc}
\hline Peptide & $I_{50}(\mu \mathrm{M})$ & MIC $(\mu \mathrm{M})$ \\
\hline Nisin & $0.12 \pm 0.01$ & 0.25 \\
Geobacillin I & $0.56 \pm 0.01$ & 1.0 \\
Geobacillin-L19P & $1.00 \pm 0.03$ & 2.0 \\
Geobacillin-L19NVA & $5.70 \pm 0.08$ & 8.0 \\
Geobacillin-Dha5F/L6I & $0.45 \pm 0.09$ & 1.0 \\
\hline
\end{tabular}

Abbreviation: Dha, dehydroalanine.

values for nisin at $0.3 \mu \mathrm{M}$ compared with $0.6 \mu \mathrm{m}$ for geobacillin I (Figure 2b and Supplementary Figure S3).

Previously, site-saturation mutagenesis was performed on the amino acids in the linker between the $\mathrm{C}$ and $\mathrm{D}$ rings of nisin. The antibiotic activities of the nisin mutants N20P, M21V, K22T and K21A were increased against several pathogenic bacteria. ${ }^{23}$ Conversely, introduction of a double Pro in this region (N20P/M21P) strongly decreased pore formation; ${ }^{17}$ this double mutant as well as the single M21P mutant also had strongly reduced antimicrobial activities. ${ }^{17,23}$ To evaluate how such mutations would affect the activity of geobacillin I, site-directed mutagenesis was used in this study to replace the naturally occuring Leu19 in geobacillin I with Pro and to introduce the tripeptide AsnValAla as linker between the C and D rings, thus generating a linker sequence that combines two of the mutations in the nisin variants with improved activity. These analogs were generated by co-expression of mutants of the precursor peptide GeoAI with the modification enzymes GeoB and GeoC in Escherichia coli as previously reported for the production of wild-type geobacillin I (Supplementary Figure S4; Tables S1 and S2) ${ }^{18}$ Compared with wildtype geobacillin I, the analogs with NVA and $\mathrm{P}$ as the linker between the $\mathrm{C}$ and $\mathrm{D}$ rings had eightfold and twofold increased MIC values, respectively (Table 1 ). The ability to induce pore formation by these analogs was also investigated. Although the efficiency of pore formation was strongly reduced, replacement of Leu19 with Pro did not abolish this activity (Figure 2b). Introduction of the amino-acid residues NVA in this region also greatly reduced the formation of pores in the bacterial cell membrane by geobacillin I. Thus, mutations in the linker peptide between the $\mathrm{C}$ and $\mathrm{D}$ rings affect the activities of nisin and geobacillin I quite differently, suggesting that the detailed mechanism of pore formation by geobacillin I differs from that of nisin. These findings also suggest that the structure of the C-terminus of class I lantibiotics may vary significantly while retaining pore formation activity.

The ability of geobacillin I to bind lipid II was investigated next using in vitro inhibition of the transglycosylation reaction catalyzed by penicillin-binding protein $1 \mathrm{~b}$ from $E$. coli. Penicillin-binding protein 1b uses lipid II as a substrate for glycan polymerization. ${ }^{24}$ Geobacillin I inhibited Penicillin-binding protein 1b-catalyzed peptidoglycan formation using $4 \mu \mathrm{M}$ heptaprenyl lipid II with a half-maximal inhibitory concentration $\left(\mathrm{IC}_{50}\right.$ ) of $4.6 \pm 0.8 \mu \mathrm{M}$ (Figure 2c). For comparison, inhibition by nisin under the same conditions displayed an $\mathrm{IC}_{50}$ of $2.9 \pm 0.6 \mu \mathrm{M}$ (Figure $2 \mathrm{c}$ ). Thus, the inhibitory activity of the two peptides is very similar.

Geobacillin I has higher stability at physiological $\mathrm{pH}$ compared with nisin. ${ }^{18}$ Although higher stability for a compound from a thermophile is not unexpected, the higher stability was somewhat surprising because nisin degradation at neutral $\mathrm{pH}$ is believed to be caused by non-enzymatic hydrolysis at Dha $5,{ }^{25}$ a residue that is also present in geobacillin I. We wondered whether the stability and hence antimicrobial activity of geobacillin I could be further improved by mutation of Dha5 in light of a previous report that the nisin analog I4K/Dha5F/L6I had higher antimicrobial activity against various bacteria. ${ }^{26}$ Geobacillin I already has a Lys at position 4, and hence the mutant Dha5F/L6I was generated; however, it proved to be only slightly more stable than the wild-type geobacillin I (Supplementary Figure S4) while displaying similar MIC values (Table 1). 
a

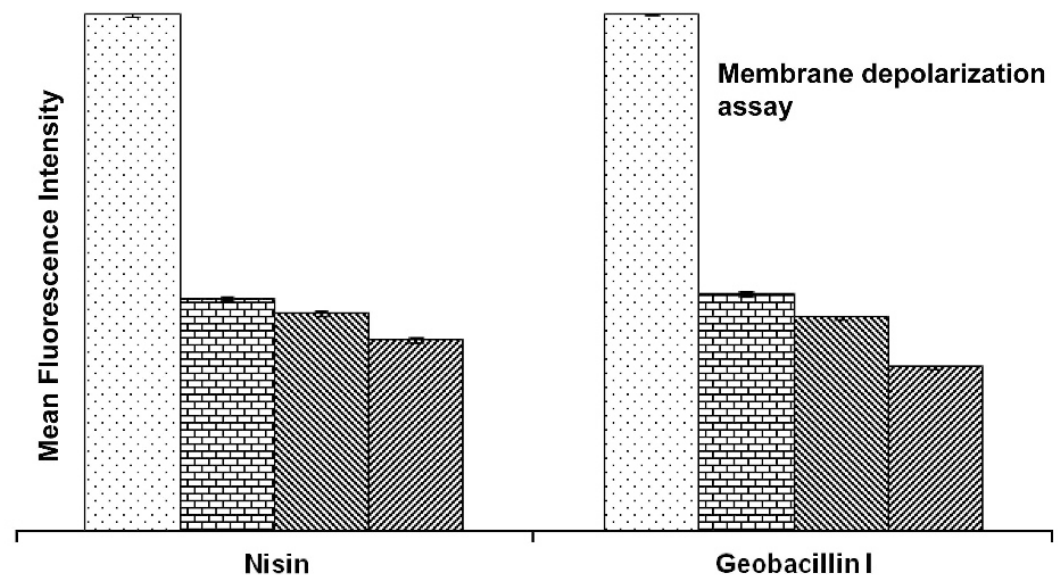

b
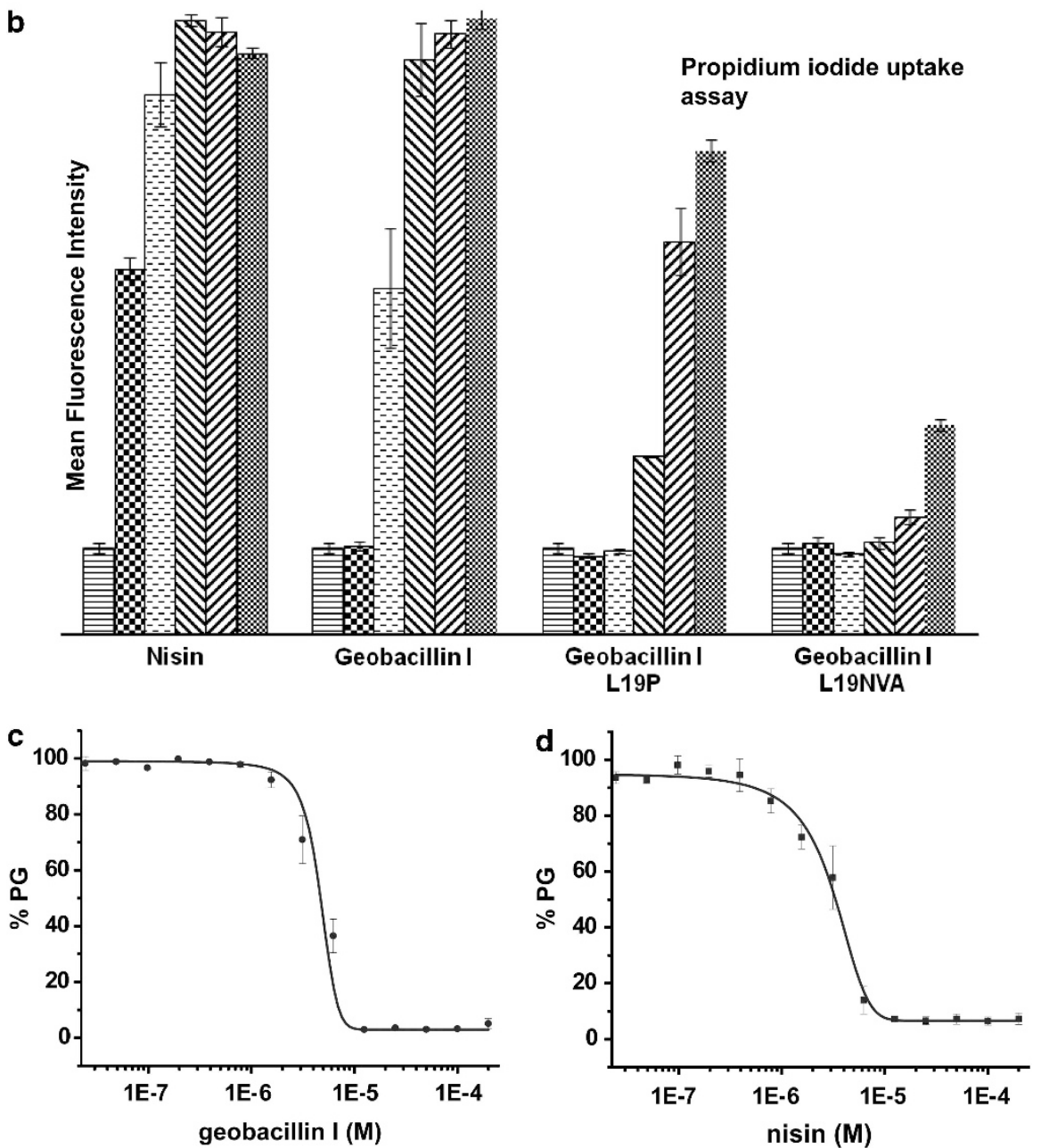

Figure 2 The effect of geobacillin I and nisin on the membrane integrity of B. subtilis ATCC 6633. (a) Average mean fluorescence intensity (MFI) of triplicate flow cytometry measurements with different concentrations of nisin and geobacillin I using 3,3'-diethyloxacarbocyanine iodide as the indicator of membrane potential. The different bars represent concentrations of 0, 0.2, 2 and $20 \mu \mathrm{m}$ (left to right). For a representation using the ratio of fluorescence at 610 and $530 \mathrm{~nm}$, see Supplementary Figure S2. (b) Increase in MFI resulting from propidium iodide uptake by B. subtilis ATCC 6633 in response to treatment with nisin and geobacillin I (average of three measurements). The different bars represent concentrations of $0,0.31,1.25,2.5,5$ and $10 \mu \mathrm{m}$ (left to right). (c) Inhibition of penicillin-binding protein $1 \mathrm{~b}$ (PBP1b)-catalyzed peptidoglycan (PG) formation by geobacillin I and by nisin (d) at a lipid II concentration of $4 \mu \mathrm{m}$ and a PBP1b concentration of $100 \mu \mathrm{m}$. Error bars represent the s.d. from triplicate experiments.

Compared with nisin, ring $\mathrm{C}$ of geobacillin $\mathrm{I}$ is reduced in size by one amino acid, the region between rings $\mathrm{C}$ and $\mathrm{D}$ is reduced in length by two amino acids and ring $\mathrm{E}$ is a lanthionine ring as opposed to a methyllanthionine ring (Figure 1c). Furthermore, geobacillin I contains two additional thioether bridges at its C-terminus. However, the antimicrobial activity and the overall mode of action of the two 
lantibiotics appear to be quite similar: binding to lipid II and pore formation.

\section{ACKNOWLEDGEMENTS}

This work was supported by the National Institutes of Health (R01 GM58822 to WAV and R01 GM076710 to SW). CGG was supported by the National Institutes of Health under Ruth L Kirschstein National Research Service Award 5 T32 GM070421 from the National Institute of General Medical Sciences.

1 Knerr, P. J. \& van der Donk, W. A. Discovery, biosynthesis, and engineering of lantipeptides. Annu. Rev. Biochem. 81, 479-505 (2012).

2 Schnell, N. et al. Prepeptide sequence of epidermin, a ribosomally synthesized antibiotic with four sulphide-rings. Nature 333, 276-278 (1988).

3 Delves-Broughton, J., Blackburn, P., Evans, R. J. \& Hugenholtz, J. Applications of the bacteriocin, nisin. Antonie van Leeuwenhoek 69, 193-202 (1996).

4 Lubelski, J., Rink, R., Khusainov, R., Moll, G. N. \& Kuipers, O. P. Biosynthesis, immunity, regulation, mode of action and engineering of the model lantibiotic nisin. Cell Mol. Life Sci. 65, 455-476 (2008).

5 Brumfitt, W., Salton, M. R. \& Hamilton-Miller, J. M. Nisin, alone and combined with peptidoglycan-modulating antibiotics: activity against methicillin-resistant Staphylococcus aureus and vancomycin-resistant enterococci. J. Antimicrob. Chemother. 50, 731-734 (2002).

6 Goldstein, B. P., Wei, J., Greenberg, K. \& Novick, R. Activity of nisin against Streptococcus pneumoniae, in vitro, and in a mouse infection model. J. Antimicrob. Chemother. 42, 277-278 (1998).

7 Mota-Meira, M., LaPointe, G., Lacroix, C. \& Lavoie, M. C. MICs of mutacin B-Ny266, nisin A, vancomycin, and oxacillin against bacterial pathogens. Antimicrob. Agents Chemother. 44, 24-29 (2000).

8 Breukink, E. \& De Kruijff, B. Lipid II as a target for antibiotics. Nat. Rev. Drug Discov. 5, 321-332 (2006).

9 Schneider, T. \& Sahl, H. G. Lipid II and other bactoprenol-bound cell wall precursors as drug targets. Curr. Opin. Investig. Drugs 11, 157-164 (2010).

$10 \mathrm{Kramer}, \mathrm{N}$. E. et al. Increased D-alanylation of lipoteichoic acid and a thickened septum are main determinants in the nisin resistance mechanism of Lactococcus lactis. Microbiology 154, 1755-1762 (2008).

11 Gravesen, A., Sørensen, K., Aarestrup, F. M. \& Knøchel, S. Spontaneous nisin-resistant Listeria monocytogenes mutants with increased expression of a putative penicillin- binding protein and their sensitivity to various antibiotics. Microb. Drug Resist. 7 127-135 (2001)

12 Khosa, S., Alkhatib, Z. \& Smits, S. H. NSR from Streptococcus agalactiae confers resistance against nisin and is encoded by a conserved nsr operon. Biol. Chem. 394, 1543-1549 (2013)

$13 \mathrm{Hsu}, \mathrm{S}$. T. et al. The nisin-lipid II complex reveals a pyrophosphate cage that provides a blueprint for novel antibiotics. Nat. Struct. Mol. Biol. 11, 963-967 (2004).

14 Brötz, H. et al. Role of lipid-bound peptidoglycan precursors in the formation of pores by nisin, epidermin and other lantibiotics. Mol. Microbiol. 30, 317-327 (1998).

15 Breukink, E. et al. Use of the cell wall precursor lipid II by a pore-forming peptide antibiotic. Science 286, 2361-2364 (1999).

16 Hasper, H. E. et al. An alternative bactericidal mechanism of action for lantibiotic peptides that target lipid II. Science 313, 1636-1637 (2006).

17 Wiedemann, I. et al. Specific binding of nisin to the peptidoglycan precursor lipid II combines pore formation and inhibition of cell wall biosynthesis for potent antibiotic activity. J. Biol. Chem. 276, 1772-1779 (2001).

18 Garg, N., Tang, W., Goto, Y., Nair, S. K. \& van der Donk, W. A. Lantibiotics from Geobacillus thermodenitrificans. Proc. Natl Acad. Sci. USA 109, 5241-5246 (2012).

19 Hasper, H. E., De Kruijff, B. \& Breukink, E. Assembly and stability of nisin-lipid II pores. Biochemistry 43, 11567-11575 (2004).

20 Brötz, H. \& Sahl, H. G. New insights into the mechanism of action of lantibioticsdiverse biological effects by binding to the same molecular target. J. Antimicrob. Chemother. 46, 1-6 (2000).

21 Yuan, J., Zhang, Z. Z., Chen, X. Z., Yang, W. \& Huan, L. D. Site-directed mutagenesis of the hinge region of nisinZ and properties of nisinZ mutants. Appl. Microbiol. Biotechnol. 64, 806-815 (2004).

22 Oman, T. J. \& van der Donk, W. A. Insights into the mode of action of the two-peptide lantibiotic haloduracin. ACS Chem. Biol. 4, 865-874 (2009).

23 Field, D., Connor, P. M., Cotter, P. D., Hill, C. \& Ross, R. P. The generation of nisin variants with enhanced activity against specific gram-positive pathogens. Mol. Micro biol. 69, 218-230 (2008).

24 Chen, L. et al. Vancomycin analogues active against vanA-resistant strains inhibit bacterial transglycosylase without binding substrate. Proc. Natl Acad. Sci. USA 100, 5658-5663 (2003).

25 Lian, L. Y. et al. Solution structures of nisin A and its two major degradation products determined by n.m.r. Biochem. J. 283, 413-420 (1992).

26 Rink, R. et al. Dissection and modulation of the four distinct activities of nisin by mutagenesis of rings $\mathrm{A}$ and $\mathrm{B}$ and by $\mathrm{C}$-terminal truncation. Appl. Environ. Microbiol. 73, 5809-5816 (2007).

Supplementary Information accompanies the paper on The Journal of Antibiotics website (http://www.nature.com/ja) 